DOI: https://doi.org/10.46296/yc.v5i9edespsoct.0117

\title{
NATURALIZACIÓN DE LA VIOLENCIA CONTRA LA MUJER EN LA EDUCACIÓN SUPERIOR
}

\section{NATURALIZATION OF VIOLENCE AGAINST WOMEN IN HIGHER EDUCATION}

\author{
Reyes-Tomalá Brenda Amparito 1; Rivadeneira-Cabrera Melany Dayana 2; León-Valle \\ Benjamín Wilson ${ }^{3}$; Vera-Meza Katty ${ }^{4}$ \\ ${ }^{1}$ Universidad Estatal Península de Santa Elena. Santa Elena, Ecuador. Correo: \\ breyes@upse.edu.ec. ORCID ID: https://orcid.org/0000-0001-6815-8507
}

2 Universidad Estatal Península de Santa Elena. Santa Elena, Ecuador. Correo: melany.rivadeneiracabrera@upse.edu.ec. ORCID ID: https://orcid.org/0000-00029758-6719

${ }^{3}$ Universidad Estatal Península de Santa Elena. Santa Elena, Ecuador. Correo: bleon@upse.edu.ec. ORCID ID: https://orcid.org/0000-0002-3374-5207

${ }^{4}$ Universidad Estatal Península de Santa Elena. Santa Elena, Ecuador. Correo: kvera@upse.edu.ec. ORCID ID: https://orcid.org/0000-0003-4849-1948

\section{Resumen}

La violencia contra las mujeres es una situación que en los últimos años se ha venido visualizando, las estadísticas en el Ecuador muestran que 1 de cada 3 mujeres sufren algún tipo de violencia, estos hechos repetitivos indican que existe un problema estructural, por lo tanto, son acciones que se manifiestan en la cotidianidad, que en algunos casos son situaciones que se viven como algo natural. La naturalización de la violencia contra la mujer tiene su génesis en los procesos históricos como resultado de las interacciones sociales y por la ausencia de un marco constitucional incluyente, es decir, que incluya a la mujer en la vida pública, la ilusión según políticas públicas que tratan de enmendar este error por medio de la participación de las instituciones de la sociedad civil particularmente las instituciones de educación superior En este sentido, la investigación tiene el objetivo de identificar las diferentes formas en la que se ha naturalizado la violencia contra la mujer en las instituciones de educación superior, con este fin se elaboró en el diseño metodológico orientado desde el enfoque mixto; cuali-cuantitativo, con un fuerte componente descriptivo la población objeto de estudio fueron los estudiantes universitarios, para evitar algún sesgo se trianguló la información con los datos de una encuesta aplicada por la SENESCY, cuyos resultados se presentan a continuación.

Palabras claves: mujer, violencia, género, educación.

\begin{abstract}
Violence against women is a situation that has been seen in recent years, statistics in Ecuador show that 1 in 3 women suffer some type of violence, these repetitive events indicate that there is a structural problem, therefore, they are actions that are manifested in everyday life, which in some cases are situations that are experienced as something natural. The naturalization of violence against women has its genesis in historical processes because of social interactions and the absence of an inclusive constitutional framework, that is, that includes women in public life, the illusion according to public policies that try to correct this error through the participation of civil society institutions, particularly higher education institutions. In this sense, the research aims to identify the different ways in which violence against women has become naturalized in institutions of higher education, for this purpose it was elaborated in the methodological design oriented from the mixed approach; quali-quantitative, with a strong descriptive component, the population under study were university students. To avoid any bias, the information was triangulated with data from a survey applied by SENESCY, the results of which are presented below.
\end{abstract}

Keywords: woman, violence, gender, education.

Información del manuscrito:

Fecha de recepción: 02 de julio de 2021.

Fecha de aceptación: 01 de octubre de 2021.

Fecha de publicación: 04 de octubre de 2021. 


\section{Introducción}

El incremento de feminicidios en el país visibiliza una situación que se encuentra presente en la sociedad esta situación afecta al desarrollo integral y armónico del tejido social, sin embargo los hechos consecutivos de violencia, lo único que muestran es la naturalización de esta contra la mujer como resultado de un proceso histórico producto de una ruptura sociocultural completamente contradictorio a los valores étnicos originarios este quiebre social produce un desequilibrio en la relación de poder en el que se relega a la mujer a un rol de inferioridad lo que produce asimetrías sociales con relación a las mujer. Consecuentemente la naturalización de la violencia que en forma primigenia como resultante de la construcción social por la incorporación de habitus; sistema de obrar, pensar, sentir asociados a la posición socia, principios generadores y organizadores de prácticas y representaciones que pueden estar objetivamente adaptadas en un fin (Boudieu, 2007). Por otro, lado el marco legal constitucional ha sido excluyente, desde la formación de la República los movimientos libertarios que propugnaron la emancipación de los territorios del yugo español relegaron a indígenas negros y mujeres de este derecho, excluyendo funcional y estructuralmente de la vida pública, la exclusión como otra expresión de violencia institucionalizada, en este contexto se evidencia la violencia como una expresión natural.

Las acciones de violencia han rebasado la racionalidad y las normas de convivencia social cosificando a las mujeres con un título de propiedad o como un bien suntuario al servicio del hogar. Por lo tanto, romper con este paradigma ha significado para algunas la muerte, ante el aumento de los casos de violencia, la intervención en el sistema de educación con buenas prácticas se volvió un imperativo, a través de enseñanzas que rompan la hegemonía de la masculinidad. con protocolos de prevención y actuación en casos de acoso, discriminación, violencia basada en género, $u$ orientación sexual.

\section{La violencia y su naturalización}

Definir la violencia no es una tarea muy fácil, sin embargo, se la realizó desde la perspectiva de algunas 
disciplinas como la del derecho que con relación a la violencia indica que significa: coacción, palabra que proviene del latín "violentia" que está relacionada con la agresividad que es un elemento biológico (Diccionario Etimológico, 2019). En esta línea de pensamiento, en el Diccionario Panhispánico del español jurídico con respecto a violencia indica que: "es el acto jurídico, fuerza intrínseca sobre un sujeto para imponerle a realizar un acto a la fuerza y a la que no puede resistir (Enciclopedía Jurídica , 2020). Además, la Organización Mundial de la salud define la violencia como el uso intencional de la fuerza física, amenaza contra uno mismo, otra persona, otro grupo, o una comunidad, que tiene consecuencias o es muy probable que tenga como un traumatismo, daños psicológicos problemas de desarrollo o hasta la muerte (OMS, 2020).

Por otro lado, la Asamblea General de las Naciones Unidas en 1993 indica como violencia hacia la mujer; todo acto basado en la pertenencia al sexo femenino que tenga o pueda tener como resultado un daño, sufrimiento físico, sexual, o psicológico para la mujer, así como las amenazas, la coacción, o la privación arbitraria de la libertad (ONU, 1993). El impacto a nivel psicológico en la víctima de violencia va a depender del tiempo al que sea sometida a la violencia y de la profundidad / intensidad y fuerza del sometimiento y a las acciones que las acciones que le sean ejercidas. Todas estas prácticas de violencia tienen un impacto en el desarrollo social, sexual y afectivo de la víctima (Cuervo \& Martínez, 2013). La violencia de género como temática es relativamente nueva y existen pocos estudios y publicaciones que muestren cifras reales y actuales de esta problemática a nivel regional y nacional sin embargo en el contexto del Ecuador se han logrado realizar avances desde la palestra pública, enfocados en abordar esta temática Con iniciativas como: la creación de unidades judiciales especializadas en violencia de género y familia, modificación del marco normativo, diseño de planes nacionales contra la violencia de género, levantamiento de encuestas nacionales, que han permitido conocer y dimensionar esta temática a través de cifras oficiales. La violencia de género sigue un ciclo que en algunos casos 
terminan en la muerte de la mujer por calidad de ser mujer. En agosto del 2019 aproximadamente 62 mujeres fueron víctimas de femicidio lo que evidencia que los niveles de violencia de género en el país son alarmantes (Aldea, 2019).

Esta problemática social trasciende etnias religiones condiciones sociales y culturales siendo así una temática de gran interés en los ámbitos públicos y privados y que tienen gran impacto en la familia, que se considera el núcleo de la sociedad. Es por esto, que se vuelve importante y necesario poder plantear propuestas y líneas de debate respecto a la violencia de género en los diferentes contextos educativos, sociales y las diferentes localidades del país, particularmente, en las instituciones de educación superior, que son las que se encuentran encargadas de formar a profesionales ciudadanos y ciudadanas que se encuentren asumiendo con responsabilidad cada una de sus acciones.

La violencia entre géneros principalmente de un hombre hacia una mujer se fundamenta en la premisa del esquema patriarcal en las relaciones interpersonales de pareja y la idea central del patriarcado. Se puede inferir como la representación de la masculinidad a través del dominio sobre las mujeres. El concepto de violencia de género es relativamente nuevo, debido a que, el trato con características violentas hacia las mujeres, no eran consideradas como violencia debido a que históricamente el hombre ha sido considerado superior a la mujer y ejercido su predominancia física sobre ella, ajustándola a distintos niveles de violencia y sometimiento, una forma naturalizada de las relaciones interpersonales en el tejido social (Amores, Rodrigo, Donoso, \& Márquez, 2021).

En este contexto, la violencia contra la mujer constituye una manifestación de la relación de poder históricamente desigual, esto deja soslayada a la mujer subordinándola a una cultura de cosificación y vulneración de su condición. En el contexto ecuatoriano históricamente la participación de las mujeres no ha sido muy destacada pero tampoco ha sido nula puesto que muchas mujeres a pesar de la opresión propia de la época, con un marco constitucional que limitaba sus 
acciones, lograron posicionarse en entornos con una compleja dinámica machista. El nombre de Matilde Hidalgo Navarro aún suena en las aulas universitarias como un referente de lucha por el reconocimiento de los derechos de las mujeres, su lucha aguerrida, constante, valiente, dio sus frutos para que hoy la mujer ecuatoriana goce de derechos entre los que se encuentran el ejercer el voto, el acceso a la educación superior, entre otros, que fueron la base para tener oportunidades para la profesionalización e inserción en el quehacer productivo. Sin embargo, y a pesar de los adelantos, que significa una progresión de derechos, paralelamente es un espacio de vulneración de estos, que constantemente se convierte en un indicador social adverso para la convivencia social. Las estadísticas presentadas por las instituciones y organizaciones a nivel nacional e internacional, como la OIT, CEPAL, ONU - Mujeres, entre otras instituciones indican la desigualdad que existe entre el hombre y la mujer. Para la CEPAL la desigualdad se manifiesta en diversos ámbitos especialmente en la educación (CEPAL, 2016).
Para poder reducir las desigualdades en torno a la educación, se abre oportunidades las cuales a su vez son sesgadas por la estructura patriarcal, la desigualdad, y las condiciones estructurales, que se van afianzando en el tejido social, es decir; social cultural. Al respecto Reche manifiesta qué: se vuelve doloroso y despiadado reconoceré que esta arma, la educación, haya sido con saña utilizada para violentar a las mujeres en el ejercicio de las relaciones de poder (Reche , 2013).

La esencia de la educación permite una renovación paradigmática en el plano cognitivo que se exteriorizan con las acciones de los individuos en sociedad. Los estudios universitarios constituyen una etapa muy importante en la vida de una persona puesto que a través de este se va formando y afirmando un proyecto de vida, desde una perspectiva holística. No obstante, se evidencia una incidencia en la interrupción del proceso educativo en las mujeres, debido a múltiples motivos como por ejemplo; el entorno familiar, las condiciones socioculturales, que privilegia la línea patrilineal al estudio del varón y las diversas condiciones 
de una relación asimétrica que desde luego para la mujer es adversa. Por otro lado, la situación se agrava cuando la estudiante está embarazada, puesto que el sistema la coloca al margen de las diferentes actividades, por su condición. Esta es otra forma de violencia contra la mujer que se han naturalizado.

A pesar de la existencia de un marco legal en el sistema de educación superior y en las instituciones que son encargadas de operativizar las leyes o normativas para que sean consecuentes con la situación, han sido pocos los esfuerzos por cambiar esta realidad como se evidencia a través de informes presentados por los organismos pertinentes. Por lo tanto, no se debe tomar como situaciones aisladas, como parte de una estadística, un número, una cifra, como parte de un indicador.

\section{La violencia una construcción social}

la Declaración de los Derechos Humanos en 1948 incorpora como parte de los convenios internacionales planes y programas de erradicación de la violencia de género. Sin embargo, poco se materializa y casi no surte el efecto deseado; qué es el modificar este tipo de conductas que afecta a las mujeres, poque están tan naturalizadas que han invadido todas las instancias de la organización civil, incluyendo las instituciones de educación superior, así lo demuestran las estadísticas. La problemática puede pasar desapercibida debido a lo natural que puede presentar una conducta inapropiada en la relación hombre y mujer, consciente 0 inconscientemente hay un reconocimiento de las acciones y la naturalización de las cosas.

Estas conductas no eran propias de los pueblos originarios, por lo que es pertinente hacer referencia al proceso colonizador que ha transitado el territorio, su población tiene bien marcado los rasgos del mestizaje, heredada por la cultura Ibérica. Desde la perspectiva antropológica se entiende como cultura; a las ideas, pensamientos, sentimientos, creencias, que consolidan la identidad de los pueblos en su reproducción social, por lo tanto, la herencia cultural recibida está plasmada por las situaciones y condiciones de la sociedad española, particularmente 
en la relación hombre - mujer y que a través de procesos de endoculturación se ha transmitido de generación en generación, hasta adquirir la forma en que hoy se manifiesta, como parte de un constructo histórico.

La ruptura de la cohesión social se manifiesta en la brecha cultural ocasionada por esta comunidad que sometieron todo tipo de resistencia, impusieron sus leyes, su forma de vida reemplazó a la originaria. Sin embargo, evidencias antropológicas y arqueológicas refieren a la existencia de culturas en el Ecuador en las que la mujer gozaba de reverencia de carácter simbólico ancestral como parte de la expresión de la vida misma. La cultura Valdivia conocida científica y culturalmente como una de las más antiguas del continente americano. Gómez en su construcción antropológica, a través de los hallazgos de la cerámica se observa objetivamente un tributo a la supremacía femenina que evidenciaba la existencia de un período matriarcal como evidencia de que la mujer actuó en espacios preponderante en el desarrollo de la civilización y que por medio de estos procesos de asimilación cultural el linaje patrilineal fue marcando la supremacía (Gómez , 2017).

En la construcción del imaginario Social en los relatos de la conquista se evidencia el carácter de los conquistadores, por lo tanto, la violencia en términos generales formaba parte de su habitus es decir de su forma de vida. Bourdieu, citado por Capdeielle con relación a este manifiesta que son disposiciones socialmente adquiridas que mueven a los individuos a vivir de una manera similar a la de otros miembros del grupo social la continuidad de la experiencia está dada por las primeras experiencias que condicionan o estructuran las siguientes es decir el habitus cómo lo socialmente encarnado y socializado en la comunidad (Capdevielle, 2011). Desde estas reflexiones como punto de partida la violencia se encuentra presente en la cotidianidad y se manifiesta en diferentes formas en ocasiones tan imperceptibles con acciones de la cotidianidad.

En investigaciones recientes España es afectada por el mismo problema y dilemas que enfrenta el Ecuador con relación a la violencia de género en los sistemas de educación superior, 
la Universidad Complutense de Madrid en colaboración con la Delegación del Gobierno para la violencia de género del Ministerio de Sanidad, Servicios Sociales e Igualdad en el 2010, realizaron un estudio respecto a la igualdad y prevención de la violencia de género en adolescentes; afirman que el $14.3 \%$ de las mujeres españolas han sufrido violencia de género. Según Díaz María José. este dato es muy importante para hacer una correlación a la investigación (Díaz , Aguado, \& Carvajal , 2010).

En el contexto ecuatoriano, El Instituto nacional de estadística y censo (INEC) afirma que 6 de cada 10 ecuatorianos han sufrido algún tipo de violencia de género la información citada corresponde a los datos recogidos por el INEC en el año 2010 y que después de 10 años se puede suponer que por medio de las políticas implementadas por el Estado, esta situación ha disminuido sin embargo los datos presentados por la SENESCYT en el 2018 indica lo contrario.

\section{La violencia un paliativo constitucional}

La Constitución del Ecuador señala que las mujeres embarazadas pertenecen al grupo de atención prioritaria, por lo tanto, los establecimientos de educación superior deben estar preparados para atender las necesidades particulares de las mujeres en estado de gestación. Sin embargo, los establecimientos de educación superior omiten tales preceptos debido a que las instalaciones no brindan condiciones necesarias para la accesibilidad; aulas ubicadas en los pisos superiores o irregulares, sin bancas diseñadas ergonómicamente con dimensiones para albergar un cuerpo con medida que se va desarrollando como conforme avanza el embarazo de las estudiantes universitarias en periodo de gestación, condiciones institucionales que deben ser mejoradas para que la mujer estudiante pueda sentirse cómoda durante su formación profesional en el proceso enseñanza aprendizaje.

Del mismo modo existe una omisión de regulaciones que orientan la implementación del centro de cuidado infantil en función del 
número de mujeres trabajadoras, asimismo en el mundo de la educación superior existen vivencias como el del estudiante que con su hijito para no perder el examen o una exposición u otra actividad estos escenarios abren espacios para que se ponga de manifiesto el desprecio social a la situación materna lo que se torna más grave para las mujeres universitarias quienes están más propensas a la deserción del sistema universitario por causas del embarazo.

Con base a esta realidad se han abierto líneas de investigación en torno a la violencia de género, las que buscan determinar las causas y efectos de este tipo de comportamiento social. Sin embargo, los esfuerzos resultan insuficientes, debido a que son recurrentes los casos de acoso en aulas universitarias, muchos de estos no se evidencian por la ausencia de la denuncia y por el silencio de las mujeres agredidas. En otros casos; los protocolos son muy flexibles que no promueven el seguimiento ni tampoco brindan soluciones prácticas y emergentes dado que el acosador solo recibe un llamado de atención volviendo al entorno en el que se originó tal desequilibrio, dejando a la víctima a un lado sin una reparación psicológica, emocional y social. Hasta ahora no se ha dimensionado el daño psico emocional en la autoestima e inseguridad causada por el acoso. Al respecto García citando a Nielsen en 1986 manifiesta que ante estas atrocidades se debe tomar partido, puesto que el silencio estimula al verdugo y la posición neutral ayuda siempre al opresor nunca a la víctima (García , 2019).

En este contexto surgen iniciativas para tratar de erradicar este problema social que como se evidencia se ha enquistado en todas las instituciones de la sociedad civilmente organizada, para este caso las instituciones de educación superior en Ecuador se reunieron para presentar una propuesta a través de la Secretaría Nacional de Educación Superior Ciencia y Tecnología e Innovación (SENESCYT). La Secretaría Nacional de Planificación y Desarrollo (SENPLADES), El Consejo de Evaluación Acreditación y Aseguramiento de la Calidad de la Educación Superior (CEAACES), EI Consejo Nacional para la Igualdad 
de Género y la agencia de las Naciones Unidas para la Igualdad de Género y el empoderamiento de las mujeres ONU mujeres, con la participación de 43 universidades ecuatorianas, en este contexto constituyeron la Red de Educación Superior y Género del Ecuador, la red académica se creó con el objeto de erradicar la discriminación y la violencia de género y aportar en la transversalización el principio de igualdad y no discriminación por razón de género. En este sentido el sistema de educación superior se constituye como un espacio de coordinación y cooperación que permite construir fortalecer $y$ potenciar acciones orientadas a promover la igualdad formal $y$ material entre mujeres y hombres. Además de construir y fortalecer la diversidad de género (SENESCYT, 2015). En este contexto, la cooperación interinstitucional permitió construir y fortalecer espacios de encuentro como vía para que estos abordajes a nivel nacional dejen de ser un tabú, se reflexione y se concientice en este proceso de interrelación y de encuentro entre los géneros.
Los resultados en la red se publicaron en el 2018 con el informe de la encuesta realizada por la Secretaría de Educación Superior Ciencia y Tecnología Innovación y la Red de Educación Superior y Género, la red en el 2018 a través del informe evidencia que la violencia de género es un flagelo que afecta a más del $60 \%$ de las mujeres de éstas el $61.4 \%$ corresponde al área urbana y el $58.7 \%$ a la Rural. La encuesta visibiliza otro dato que se debe destacar; una de cada cuatro mujeres ha vivido violencia sexual, además, el $53.9 \%$ ha vivido violencia psicológica. El mismo informe indica que el acoso y la violencia contra la mujer a nivel universitario tienen diferentes aristas, las formas de violencia se generan en el marco de las relaciones de pareja, acorde con el grupo etario. Es decir entre estudiantes supuestamente en el marco igualitario. Por otro lado, el informe indica que las mujeres estudiantes sufren el acoso y el abuso sexual por parte de docentes y administrativos. Estos actos están marcados por la relación asimétrica de poder (SENESCYT, 2018: 4). 
La carencia de denuncia y la naturalidad de esta situación hace que en la actualidad esta problemática quede en la impunidad, puesto que, las circunstancias son complejas de comprobar porque se disfraza las acciones, con relaciones consensuadas, cuando la comunidad acepta un hecho reprobado, sus cimientos morales se debilitan.

Desde el punto de vista de Foucault presentado por Ávila en el 2006; el poder desde los operadores de dominación no es algo que posee, sino que, se ejerce. Lo que supone un carácter relacional y de un desequilibrio. Desde esta perspectiva cabe la pregunta de cómo se construyen las relaciones de sometimiento ante el abuso (Ávila, 2006). De ahí que el poder sea el resultado de relaciones sociales asimétricas que se pueden dar en cualquier ámbito, esta comprensión es importante para explicar las causas de las distintas formas de violencia que se ejercen contra la mujer a lo largo de la vida en el ámbito de la familia, la comunidad, en las relaciones personales, laborales, en la esfera pública y privada.
Es importante resaltar que mediante resolución RPC-SO-20.No.3012018 el Consejo de Educación Superior presenta el protocolo de prevención y actuación en caso de acoso discriminación y violencia basada en género orientación sexual en las instituciones de educación superior se exhorta a todas las universidades aplicar dicho protocolo contra la violencia de género y discriminación (CES, 2019) Este documento estandariza criterios convirtiéndose en una base para la generación de normativas en cada universidad. delegando a la autonomía universitaria el tratamiento de esta problemática ante un escenario en el que las mujeres víctima de violencia de género, se ve en medio de procesos disciplinarios tendientes a su revictimización en lo que aquello que en materia penal se denomina "ius puniendi", para referirse a las normas jurídicas penales y derecho penal, que contempla la aplicación de una sanción, por lo tanto, el derecho se encarga de regular las actividades de las personas que viven en sociedad. En este caso se pone de manifiesto en las universidades para los profesores sometidos a procesos de sanción en 
los que la víctima parece como testigo en los que el sistema se siente satisfecho con una sanción escrita, pecuniaria, o en el mejor de los casos, la separación del agresor de su cargo docente sin que haya una efectiva restitución, efectivizada para la persona que fue vulnerada.

\section{Metodología}

El diseño metodológico para la investigación parte del enfoque cualicuantitativo, desde la perspectiva de Hernández es pertinente para cuantificar y cualificar el objeto de estudio (Hernández \& et al , 2016). En el caso de la investigación la naturalización de la violencia contra la mujer en la educación superior es presentar y visibilizar a la violencia como una situación estructural, de modo que se encuentra arraigado en todo nivel de la sociedad y que algunos hechos inofensivos, pueden ser actos de violencia, socializado tanto que por el tejido social son pensados como naturales.

La población objeto de estudio está conformada por estudiantes universitarios se aplicó un muestreo no probabilístico por conveniencia este tipo de muestreo se utiliza cuando no se dispone de los sujetos objeto de investigación; por su accesibilidad y proximidad. Este tipo de instrumento se usa también cuando la población es demasiado grande y resulta imposible incluir a cada individuo. Para la medición se usó los criterios de validez y de confiabilidad; para este fin se utilizó el método Delphi. El muestreo se aplicó a estudiantes que voluntariamente respondieron el cuestionario.

Para la recolección de datos se envió el instrumento a los estudiantes en la Universidad vía correo electrónico para que procedan a responder la encuesta de manera voluntaria el número de estudiantes que respondió la encuesta fue de 368 estudiantes, que como se explicó, constituye una muestra por conveniencia. Adicionalmente, se contrastó la información con las estadísticas presentadas por las IES, con el fin de brindar mayor fundamentación a la investigación a través del proceso de triangulación el tipo de estudio por la temporalidad es transversal. 


\section{Resultados y discusión}

De los ítems que conforman el instrumento para la recolección de datos; la encuesta, los autores han seleccionado las que se presentan a continuación cabe indicar que estas preguntas presentan características que permiten fundamentar con mayor propiedad la investigación.

Tabla No.1 ¿A qué se atribuye con las mujeres?

\begin{tabular}{cccccc}
\hline & Indicador & Frecuencia & Porcentaje & $\begin{array}{c}\text { Porcentaje } \\
\text { válido }\end{array}$ & $\begin{array}{c}\text { Porcentaje } \\
\text { acumulado }\end{array}$ \\
\hline Válido & Cultura sexista local & 108 & 29,3 & 29,3 & 29,3 \\
& $\begin{array}{c}\text { Cultura sexista } \\
\text { universitaria }\end{array}$ & 25 & 6,8 & 6,8 & 36,1 \\
& Machismo & 235 & 63,9 & 63,9 & 100,0 \\
\cline { 2 - 6 } & Total & $\mathbf{3 6 8}$ & $\mathbf{1 0 0 , 0}$ & $\mathbf{1 0 0 , 0}$ & \\
\hline
\end{tabular}

Fuente: Estudiantes universitarios. Elaborado por: Autores.

El resultado de la encuesta con respecto a la pregunta $A$ qué atribuye la violencia contra la mujer el $63.9 \%$ de los encuestados indican que se debe al machismo. Es decir, todavía se videncia en la población, como construcción del tejido social el machista, esta conducta, comportamientos, y creencias que promueven, producen y rechazan de forma negativa la superioridad del hombre sobre la mujer lo que desemboca en violencia contra la mujer se puede inferir que este es el resultado de una herencia, denominada desde la antropología endoculturización por medio del habitus como sistema de exposición duradera y transferible como principio generadores $y$ organizadores de las prácticas y representaciones que pueden ser adaptadas marcando los estereotipos sociales de descendencia patrilineal que le han atribuido un rol sociocultural a las mujeres como ama de casa y de servicio a la familia. Esta reflexión se fundamenta con los resultados de la encuesta que indican que el $29.3 \%$ atribuye a una conducta sexista, es decir cosificando a las mujeres. 
Tabla No. 2. La violencia contra las mujeres considerada como un problema.

\begin{tabular}{cccccc}
\hline & & Frecuencia & Porcentaje & $\begin{array}{c}\text { Porcentaje } \\
\text { válido }\end{array}$ & $\begin{array}{c}\text { Porcentaje } \\
\text { acumulado }\end{array}$ \\
\hline Válido & Solo de mujeres & 10 & 2,7 & 2,7 & 2,7 \\
& $\begin{array}{c}\text { Solo de hombres } \\
\text { De ambos, hombres y } \\
\text { mujeres }\end{array}$ & 4 & 1,1 & 1,1 & 3,8 \\
& Total & $\mathbf{3 6 8}$ & $\mathbf{9 6 , 2}$ & 96,2 & 100,0 \\
\cline { 2 - 6 } & & $\mathbf{1 0 0 , 0}$ & $\mathbf{1 0 0 , 0}$ & \\
\hline
\end{tabular}

Fuente: Estudiantes universitarios. Elaborado por: Autores.

Con relación a la pregunta que se refiere a quien le concierne la violencia contra la mujer los consultados en el $92.2 \%$ indican que es un problema que corresponde al hombre y también a las mujeres, es decir; es una situación en el que interviene la pareja. Esta respuesta tiene algunas lecturas en primer lugar; la aceptación de un problema que atañe a dos y no a una sola persona, o sea, no solo le corresponde a la mujer. En segundo lugar; la visibilidad y aceptación de una realidad que es indiscutible, la violencia contra la mujer, de modo que la acción de los diferentes estamentos de la sociedad civil se constituye en una ecuación sine qua non.

Tabla No. 3. Cree usted que la condición de mujer pone en situación de vulnerabilidad a las estudiantes universitarias.

\begin{tabular}{cccccc}
\hline & & Frecuencia & Porcentaje & $\begin{array}{c}\text { Porcentaje } \\
\text { válido }\end{array}$ & $\begin{array}{c}\text { Porcentaje } \\
\text { acumulado }\end{array}$ \\
\hline Válido & $\mathrm{Si}$ & 162 & 44,0 & 44,0 & 44,0 \\
& No & 206 & 56,0 & 56,0 & 100,0 \\
\cline { 2 - 6 } & Total & $\mathbf{3 6 8}$ & $\mathbf{1 0 0 , 0}$ & $\mathbf{1 0 0 , 0}$ & \\
\hline
\end{tabular}

Fuente: Estudiantes universitarios. Elaborado por: Autores.

En referencia a la interrogante cree usted que la condición de mujer pone en situación de vulnerabilidad a las estudiantes universitarias. La respuesta a esta interrogante tiene que ver con la condición de ser mujeres, el ser mujer en una sociedad patriarcal las hace susceptible a ciertas formas de acoso. La diferencia en esta pregunta guarda una diferencia de un $6 \%$ es decir, un margen estrecho entre el criterio (si ) con relación al (no). El $44 \%$ de la población encuestada indica que la condición de ser mujer de las estudiantes 
universitarias la hace vulnerable. Mientras que el $56 \%$ indica que no.

Se realizó una correlación con la pregunta planteada a grupos de mujeres las que indicaron que es algo con lo que se tiene que aprender a lidiar, esta respuesta tiene una doble implicación por un lado aceptación o resignación a tales circunstancias, mientras que por otro lado, se puede interpretar que el problema está tan enraizado y como se ha manifestado, es estructural, lo que demuestra el grado de descomposición en el que se encuentra todo el sistema de la sociedad, de modo que estas acciones, actitudes y comportamientos se encuentra naturalizada, asimismo se contrasta con el informe de SENESCYT en el que se evidencia, que la violencia de género es un flagelo que afecta a más del $60 \%$ de las mujeres, de esto se desprende que el $61.4 \%$ corresponde al área urbana y el 58.7 $\%$ del área rural. La encuesta visibiliza otro dato que se debe destacar; una de cada cuatro mujeres ha vivido violencia sexual, además, el $53.9 \%$ ha vivido violencia psicológica.

Tabla No. 4. Considera que el entorno universitario existe violencia contra la mujer

\begin{tabular}{lccccc}
\hline & & Frecuencia & Porcentaje & $\begin{array}{c}\text { Porcentaje } \\
\text { válido }\end{array}$ & $\begin{array}{c}\text { Porcentaje } \\
\text { acumulado }\end{array}$ \\
\hline Válido & $\mathrm{Si}$ & 174 & 47,3 & 47,3 & 47,3 \\
& No & 194 & 52,7 & 52,7 & 100,0 \\
\cline { 2 - 6 } & Total & $\mathbf{3 6 8}$ & $\mathbf{1 0 0 , 0}$ & $\mathbf{1 0 0 , 0}$ & \\
\hline
\end{tabular}

Fuente: Estudiantes universitarios. Elaborado por: Autores.

La respuesta a la pregunta considera que el entorno universitario existe violencia contra la mujer, el $47.3 \%$ de la población indica que sí existe violencia contra las mujeres en todo el entorno universitario. En este sentido es importante recordar la definición de violencia que se ha desarrollado en la investigación; como el uso intencional de la fuerza física, amenaza, contra el mismo, otra persona, o a un grupo de una comunidad, que tiene consecuencias y es muy probable que tenga como consecuencia un traumatismo, daño psicológico, problemas de desarrollo, además, de la relación de poder como resultado de las interacciones sociales asimétricas se presentan con mayor 
vulnerabilidad y en condiciones más recurrentes cuando las estudiantes presentan problemas académicos y con grupos de estudiantes históricamente discriminados, particularmente la mujer. Por otro lado, el $52.7 \%$ manifiesta que considera que no existe violencia contra la mujer; evidentemente la diferencia en esta respuesta es del 4 $\%$, por lo tanto, es una problemática latente en las instituciones de educación.

Tabla No.5. Conoces si existen normativas en la Universidad tendientes a la prevención y erradicación de la violencia contra la mujer.

\begin{tabular}{cccccc}
\hline & & Frecuencia & Porcentaje & $\begin{array}{c}\text { Porcentaje } \\
\text { válido }\end{array}$ & $\begin{array}{c}\text { Porcentaje } \\
\text { acumulado }\end{array}$ \\
\hline \multirow{2}{*}{ Válido } & $\mathrm{Si}$ & 200 & 54,3 & 54,3 & 54,3 \\
& No & 168 & 45,7 & 45,7 & 100,0 \\
\cline { 2 - 6 } & Total & $\mathbf{3 6 8}$ & $\mathbf{1 0 0 , 0}$ & $\mathbf{1 0 0 , 0}$ & \\
\hline
\end{tabular}

Fuente: Estudiantes universitarios. Elaborado por: Autores.

Ante el interrogante conoce usted si existen normativas en la Universidad tendientes a la prevención o erradicación de la violencia los encuestados respondieron en un 45 $7 \%$ de forma negativa mientras que el $54.3 \%$ indica que si conoce el protocolo, lo que se puede interpretar que las instituciones de educación superior deben redoblar esfuerzos para la socialización con la comunidad académica, la normativa de protocolo de prevención y actuación en caso de acoso discriminación y violencia basada en género y orientación sexual en las instituciones de educación superior propuestos por el CES en el 2018. Por otro lado; desconocer el protocolo es desconocer los derechos de cómo actuar ante este tipo de situación, por lo que al desconocer estos derechos la situación se puede acrecentar 0 agravar en las instituciones de educación superior.

\section{Otros aspectos para considerar}

La insuficiente oportunidad para el desarrollo pleno de la mujer como profesional es otra forma de violencia la discriminación es violencia el seminario internacional de calidad de educación superior y género evidenció que el Ecuador es el país de América Latina que más invierte en educación superior donde al $2 \%$ del PIB. Este dato es apoyado 
por la información estadística del Instituto Nacional de Estadística y Censos lo que se entendería como un gran avance en lo que se refiere a la inserción de la mujer dentro del sistema educativo. La matrícula femenina supera a la matrícula masculina a diciembre del 2013 este porcentaje llegó al 52 \% frente al 48 $\%$. Para el 2019 se evidencia un incremento del $1 \%$ del ingreso de educación superior de las mujeres es decir que el $53 \%$ de ellas ingresaron al sistema de educación superior y un $47 \%$ los varones lo que indica que las mujeres están accediendo más a la educación superior en la actualidad que los varones.

\section{Conclusiones}

Para algunos pensadores retóricos ortodoxos no resultaría necesario usar falda y tacones para comprenderla problema la problemática situación por la que la violencia de género viven muchas estudiantes mujeres universitarias, el dolor la tristeza que son elementos esenciales para que desde el conocimiento dimensionar las huellas silenciosas que la violación contra la mujer arraiga la desconfianza, el miedo, el entorno académico, que con violentas y primitivas prácticas se convierten en el sece letal de un proyecto profesional que acaba incluso con las expectativas de un grupo familiar en la sociedad.

Estas reflexiones enfatizan el adagio "calificar a la violencia de género como un asunto de mujeres", es parte del problema. Escenarios como éstos han naturalizado la problemática e incluso lo han cotizado, pues asentándose en seudo ciencias hay quienes atribuyen a la comisión de los delitos sexuales a partir de la influencia motivada de la víctima a la que se le atribuye supuesta predisposición para erigir el rol y por lo tanto, ser las más culpables de dichos comportamientos lesivos

El machismo y la violencia se constituye hoy por hoy en un problema estructural en la sociedad una amenaza latente a las perspectivas de superación, profesionalización perfeccionamiento de las mujeres, fundamentadas en concepciones que omiten principios fundamentales de reconocimiento a la categoría del ser humano, desvinculada esta del sexo y aquella más radicales que 
cosifican a la mujer, de ser así las mujeres vivirán en un cuerpo "para otros".

\section{Bibliografía}

Aldea. (21 de agosto de 2019). Fundacionaldea.org.

Obtenido de https://www.fundacionaldea.o rg/noticias-aldea:

https://www.fundacionaldea.o rg/noticias-aldea

Amores, P., Rodrigo, M. J., Donoso, T., \& Márquez, M. L. (23 de enero de 2021). Fundacionlacaixa.org. Obtenido de Fundacionlacaixa.org: https://fundacionlacaixa.org/d ocuments/10280/215682/Tall er+0+Programa+Intervencio $\mathrm{n}+$ Psicosocial+para+mujeres. pdf/5e05dd7c-eb11-4680bb4f-a563b5ff1b4d

Ávila, F. (2006). El concepto de poder en Michael Foucault. Telos, 215 - 234.

Boudieu, P. (2007). El sentido Práctico. En P. Bourdieu, El Sentido Práctico (pág. 456). Buenos Aires: Siglo XXI Editores Argentina S.A.

Capdeielle, J. (20011). Concepto de Habitus. Andaluza de Ciencias Sociales.

Capdevielle, J. (02 de Febrero de 2011). Concepto de habitus con Bourdieu. Obtenido de dialnet.unirioja.es/descarga/a rticulo/3874067.pdf:

https://dialnet.unirioja.es/desc arga/articulo/3874067.pdf

CEPAL. (21 de Octubre de 2016). La Matriz de Desigualadad Social en América Latina. Obtenido de www.cepal.org/: https://www.cepal.org/sites/d efault/files/events/files/matriz _de_la_desigualdad.pdf

CES. (12 de mayo de 2019). Protocolo de prevención y actuación en caso de acoso discriminación y violencia basada en género y orientación sexual en las instituciones de educación superior. Obtenido de educacionsuperior.gob.ec/: https://www.educacionsuperi or.gob.ec/wpcontent/uploads/downloads/2 019/12/acuerdo no 201913 9_anexo_protocolo_itts.pdf

Cuervo, M., \& Martínez, J. (23 de Enero-junio de 2013). Redalyc. Obtenido de https://www.redalyc.org/pdf/1 390/139029198007.pdf: https://www.redalyc.org/pdf/1 390/139029198007.pdf

Díaz, M., Aguado, J., \& Carvajal, M. I. (2010). Igualdad y Prevención de la Violencia de Género en la adolescencia. Madrid: Centro de Publicaciones.

Diccionario Etimológico. (01 de Juio de 2019). Etimología de 
violencia. Obtenido de Etimologias.dechile.net: http://etimologias.dechile.net/ ?violencia\#: :text=

Enciclopedía Jurídica. (23 de enero de 2020).

http://www.enciclopediajuridica.com/. Obtenido de http://www.enciclopediajuridica.com/: http://www.enciclopediajuridica.com/

García, J. (2019). Código de los derechos de las víctimas. Instituto de Andaluza de Administración Pública, 11.

Gómez, R. (2017). Las culturas Valdivia y Milagro - Quevedo en la cuenca baja del rio Milagro. Milagro: Universidad Estatal de Milagro.

Hernández, R., \& et al. (2016). Metodología de la Investigación. México: Latinoamericana.

OMS. (29 de Noviembre de 2020). Who int/topic/gender_based_viole nce/es/. Obtenido de https://www.who.int/topics/ge nder_based_violence/es/: https://www.who.int/topics/ge nder based violence/es/

ONU. (20 de Diciembre de 1993). www.ohchr.org/sp. Obtenido de www.ohchr.org/sp/profession alinterest: https://www.ohchr.org/sp/prof essionalinterest/pages/violen ceagainstwomen.aspx

Reche, F. (11 de Diciembre de 2013).

www.africafundacion.org/laeducacion. Obtenido de www.africafundacion.org/: https://www.africafundacion.o rg/la-educacion-es-el-armamas-poderosa-con-la-quepuedes-cambiar-el-mundo

SENESCYT. (18 de Abril de 2015). Red para conseguir igualdad de génro en educación superior. Obtenido de red.educacionsuperior.gob.ec /una-red-para-conseguirigualdad-de-genero-eneducacion-superior/: https://www.educacionsuperi or.gob.ec/una-red-paraconseguir-igualdad-degenero-en-educacionsuperior/

SENESCYT. (2018: 4). Protocolo de actuación en caso de discriminación y violencia. Quito - Ecuador: SENESCYT. 